\title{
Factors affecting genotyping success in giant panda fecal samples
}

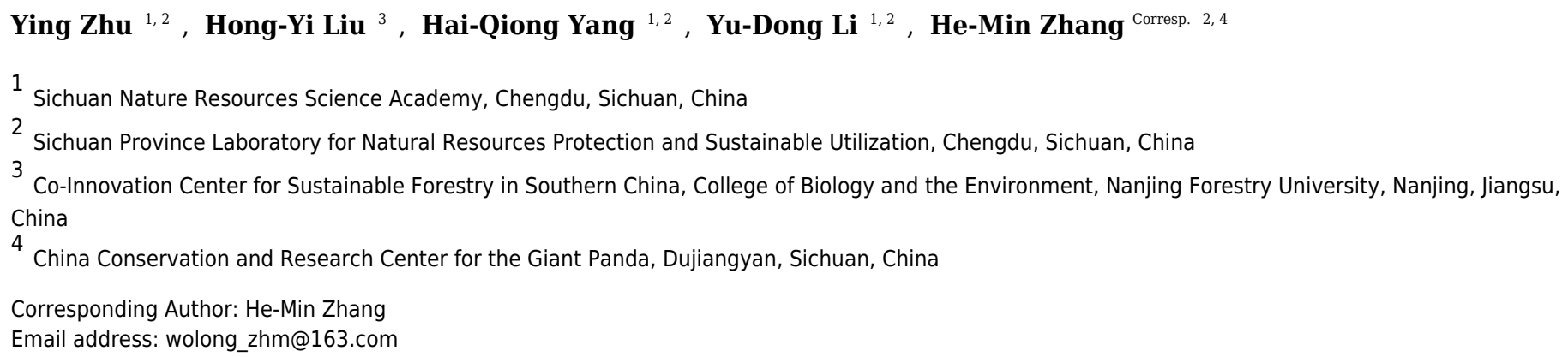

Fecal samples play an important role in giant panda conservation studies. Optimal preservation conditions and choice of microsatellites for giant panda fecal samples have not been established. In this study, we evaluated the effect of four factors, namely storage type [ethanol (EtOH), EtOH $-20^{\circ} \mathrm{C}$, 2-step storage medium, DMSO/EDTA/Tris/salt buffer (DETs) and frozen at $-20^{\circ} \mathrm{C}$, storage time $(1,3$, and 6 months), fragment length, and repeat motif of microsatellite loci, on the success rate of microsatellite amplification, and allelic dropout (ADO) and false allele (FA) rates from giant panda fecal samples. Amplification success and ADO rates differed between the storage types. Freezing was inferior to the other four storage methods based on lowest average amplification success and highest ADO rates $(P<0.05)$. The highest microsatellite amplification success was obtained from either $\mathrm{EtOH}$ or 2-step storage medium at three storage time points. Storage time had a negative effect on the average amplification of microsatellites and samples stored in $\mathrm{EtOH}$ and 2-step storage medium were more stable than the other three storage types. We only detected the effect of repeat motif on ADO and FA rates. The lower ADO and FA rate was obtained from tri- and tetra-nucleotide loci. We suggest that freezing should not be used for giant panda fecal preservation in microsatellite studies and EtOH and 2-step storage medium should be chosen on priority for long-term storage. We recommend candidate microsatellite loci with longer repeat motif to ensure greater genotyping success for giant panda fecal studies. 


\section{Factors Affecting Genotyping Success in Giant Panda Fecal Samples}

2 Ying ZHU ${ }^{1,2}$, Hong-Yi LIU ${ }^{3}$, Hai-Qiong YANG ${ }^{1,2}$, Yu-Dong LI ${ }^{1,2}$, He-Min ZHANG ${ }^{2,4^{*}}$

$3{ }^{1}$ Sichuan Nature Resources Science Academy, No. 24, the South $2^{\text {nd }}$ Section of Yihuan Road, Chengdu, 610015, 4 Sichuan Province, China.

$5 \quad 2$ Sichuan Province Laboratory for Natural Resources Protection and Sustainable Utilization, No. 24, the South $2^{\text {nd }}$

6 Section of Yihuan Road, Chengdu, 610015, Sichuan Province, China.

$7{ }^{3}$ Co-Innovation Center for Sustainable Forestry in Southern China, College of Biology and the Environment,

8 Nanjing Forestry University, No.159 Lonpan Road, Nanjing, 210037, Jiangsu Province, China

$9{ }^{4}$ China Conservation and Research Center for the Giant Panda, No. 98 Tongjiang Road, Dujiangyan, 611800,

10 Sichuan Province, China

$11{ }^{*}$ Corresponding author: He-Min ZHANG, Phone: +86-837-6246861; Fax: +86-837-6246776. Email address: 12 wolong_zhm@163.com; wolong_zhm@126.com 


\section{Abstract}

14 Fecal samples play an important role in giant panda conservation studies. Optimal preservation conditions and

15 choice of microsatellites for giant panda fecal samples have not been established. In this study, we evaluated the

16 effect of four factors, namely storage type [ethanol (EtOH), EtOH $-20^{\circ} \mathrm{C}, 2$-step storage medium,

$17 \mathrm{DMSO} / \mathrm{EDTA} /$ Tris/salt buffer (DETs) and frozen at $\left.-20^{\circ} \mathrm{C}\right]$, storage time $(1,3$, and 6 months), fragment length, and

18 repeat motif of microsatellite loci, on the success rate of microsatellite amplification, and allelic dropout (ADO) and

19 false allele (FA) rates from giant panda fecal samples. Amplification success and ADO rates differed between the

20 storage types. Freezing was inferior to the other four storage methods based on lowest average amplification success

21 and highest ADO rates $(P<0.05)$. The highest microsatellite amplification success was obtained from either EtOH

22 or 2-step storage medium at three storage time points. Storage time had a negative effect on the average

23 amplification of microsatellites and samples stored in EtOH and 2-step storage medium were more stable than the

24 other three storage types. We only detected the effect of repeat motif on ADO and FA rates. The lower ADO and FA

25 rate was obtained from tri- and tetra-nucleotide loci. We suggest that freezing should not be used for giant panda

26 fecal preservation in microsatellite studies and EtOH and 2-step storage medium should be chosen on priority for

27 long-term storage. We recommend candidate microsatellite loci with longer repeat motif to ensure greater

28 genotyping success for giant panda fecal studies. 


\section{Introduction}

31 Sampling of feces has become a feasible and widely used method for researchers to obtain genetic data in the field,

32 especially in genetic research on endangered animals. Such high usage is attributable to the sampling being

33 convenient, random, non-invasive, and non-interfering to the animal's activity, and is facilitated by the abundance of

34 samples (Kohn and Wayne 1997). The research using fecal DNA includes documents on species identification

35 (Dalen et al. 2004), sex determination (Huber et al. 2002), kinship and paternity (Constable et al. 2001), population

36 genetic diversity (Zhang et al. 2007), adaptive variation (Wan et al. 2006), population genetic structure (Zhu et al.

37 2013), dispersal pattern (Zhan et al. 2007), population size (Eggert et al. 2003), evolutionary history of species

38 (Chen et al. 2013), mating system (Garnier et al. 2001), and disease information (Zhang et al. 2012).

Despite the many advantages as stated above, low quantity and poor quality of fecal DNA, often results in the

failure of amplification and errors in microsatellite genotyping in such studies (Taberlet et al. 1996). This renders the conclusion of genetic studies unreliable and reduces the confidence in inferring such results for formulating management and conservation strategies (Pompanon et al. 2005). Several investigations suggest that careful choice of microsatellite loci and the method used for feces preservation could enhance the genotyping success and feasibility of the use of fecal samples in such studies (Broquet et al. 2007; Tende et al. 2014). preserved fecal samples from different species (reviewed in Tende et al. 2014). The conclusions have, however,

47 been inconsistent, even for the same preservation medium, suggesting that the optimal storage medium varies with

48 species, environmental conditions, and other factors (Piggott \& Taylor 2003). Moreover, it was reported that the 
50

51

52

53

54

55

56

57

58

59

60

61

62

63

64

65

66

67 storage periods in the preservation of fecal DNA of giant panda; 2) determine the optimal method that produced the

68 highest amplification success and lowest genotyping errors of microsatellites; 3) evaluate the effect of fragment

69 length and repeat unit on the genotyping success at microsatellite loci. We believe that the information gained in the

could be the reason for the varied degradation rate of fecal DNA in different preservation media. Such observations necessitate the assessment of the storage type for each new fecal study.

DNA fragment length and the microsatellite repeat motif present in it are known to greatly impact the amplification and microsatellite genotyping success (Broquet et al. 2007). Most of the studies revealed that the amplification success decreased and the genotyping error rates increased with the fragment length and that the dinucleotides were superior to longer repeat units (reviewed in Broquet et al. 2007). In contrast, other studies have reported contradictory results, demonstrating that the allele length had no effect on the error rates (Frantz et al. 2003) or that the longer fragments were easier to be amplified than the shorter ones (Whittier et al. 1999). Furthermore, motifs with longer nucleotide repeats were associated with lower error rates compared to the di-nucleotide motifs (Kruglyak et al. 1998).

The giant panda (Ailuropoda melanoleuca) is an endangered species in China owing to habitat fragmentation.

Fecal samples play an important role in its conservation studies, and they have been widely used in several genetics studies (Chen et al. 2013; Wan et al. 2006; Zhan et al. 2007; Zhang et al. 2007; Zhu et al. 2013). DNA obtained from fecal samples have been stored under dried conditions (Wan et al. 2006) and in EtOH (Zhang et al. 2004; Zhang et al. 2012). However, to our knowledge, evaluation of a long-term storage type for fecal DNA has not been conducted yet. Research on the effects of microsatellite fragment length and microsatellite motif has also been limited in giant panda fecal studies. The present study aimed to 1) compare the performance of five storage conditions and three (enth and repeat unit on the genotyping success at microsatellite loci. We believe that the information gained in the 
70 present study would be useful in guiding researchers to choose the most suitable preservation medium and

71 microsatellite loci in giant panda fecal studies and allow for assessment of results from past studies.

\section{Methods and Materials}

\section{Sample collection and preservation}

74 We obtained permission from the Conversation Base of China Research and Conservation Center for the Giant

75 Panda (CRCCGP) and the China Giant Panda Protection and Management Office to collect all the samples and

76 confirmed that we did not impact the animals during the sampling. Eighty-five fecal samples from 17 captive giant

77 pandas housed in the Dujiangyan Giant Panda Conservation Base, were collected on March 20, 2015. The samples

78 were collected from the outdoor house in the afternoon after giant pandas were recalled to their indoor house. Fresh

79 samples (<12-hours-old) were collected using disposable gloves and transferred into Ziploc bags, with the sample

80 information marked clearly on the bags. The samples were transported immediately to the laboratory after 2 hours

81 journey by car. The surface of each sample was peeled off with sterile tweezers, and the sample from each

82 individual was mixed to avoid an uneven distribution of intestinal cells (Kohn \& Wayne 1997). The samples were

83 then divided into five parts and transferred to five storage media, namely absolute $\mathrm{EtOH}$, absolute $\mathrm{EtOH}$ at $-20^{\circ} \mathrm{C}$,

84 silica after 24-hours treatment with EtOH, which was designated as the "2-step storage medium" (Tende et al. 2014),

$85 \mathrm{DMSO} / \mathrm{EDTA} /$ Tris/salt buffer (DETs) buffer (Frantzen et al. 1998), or frozen at $-20^{\circ} \mathrm{C}$. In addition, we also

86 obtained blood samples from these 17 individuals for use as reference samples when comparing the microsatellite

87 genotypes from the fecal samples. The blood samples were collected by a veterinarian during the routine medical

88 examination of pandas for disease monitoring and were stored in a $-80^{\circ} \mathrm{C}$ freezer. 
90 Two-gram dried samples were weighed from each storage medium, transferred to individual 50-mL tubes containing

$9110 \mathrm{~mL}$ Tris/NaCl/EDTA/SDS (TNES) buffer and $80 \mu \mathrm{L} 20 \mathrm{mg} / \mathrm{mL}$ proteinase $\mathrm{K}$, and incubated overnight at $65^{\circ} \mathrm{C}$ in

92 a water bath. DNA from the fecal samples was extracted using the modified phenol-chloroform extraction method

93

94

95

96

97

98

99

100

101

102

103

104

105

106

107

108

109

110 described by Wan et al. (2006). To evaluate the effect of storage time for each storage condition, DNA was

extracted after 1, 3, and 6 months, respectively. DNA from blood was extracted with the Invitrogen blood extraction

kit (Invitrogen, USA) following the protocol prescribed by the kit manufacturer. A total of 272 DNA samples

(extracted from 255 fecal and 17 blood samples) were used in the present study.

Nine microsatellite loci (Gp4, Aime10, Aime16, Panda22, Panda25, Panda29, Gpz6, Gp129, and Gpl60 (Huang et

al. 2015; Shen et al. 2007; Wu et al. 2009; Zhang et al. 2009) ) with good performance in amplification and

genotyping were chosen for evaluation in the present study. All the forward primers were mixed with a fluorescent

M13 primer (5' -CACGACGTTGTAAAACGAC-3' , Li-COR Inc., Nebraska, USA). The amplifications were carried out in a $10-\mu \mathrm{L}$ reaction mixture that contained $10 \mathrm{X}$ buffer with $25 \mathrm{mM} \mathrm{MgCl}_{2}, 0.2 \mathrm{~mol} / \mathrm{L}$ of each primer, 1

$\mu \mathrm{L}$ of $1 \mu \mathrm{M}$ Infrared Dye Phosphoramidite-labeled M13 primer, $0.2 \mathrm{mmol} / \mathrm{L}$ dNTPs, $0.25 \mathrm{U}$ r-Taq (TaKaRa Ltd, Dalian, China), and approximately $10 \mathrm{ng}$ of the DNA template. For the fecal DNA samples, $0.1 \mu \mathrm{L}$ BSA was added

to each reaction mixture. All the amplifications were performed using a touch-down profile which began at $95^{\circ} \mathrm{C}$ for $5 \mathrm{~min}$, followed by 15 touchdown cycles of $95^{\circ} \mathrm{C}$ for $30 \mathrm{~s}, 62.5-52^{\circ} \mathrm{C}$ for $30 \mathrm{~s}$, and $72^{\circ} \mathrm{C}$ for $30 \mathrm{~s}$, decreasing by $0.7^{\circ} \mathrm{C}$ to the annealing temperature and 25 cycles with an annealing temperature of $52^{\circ} \mathrm{C}$. A final amplification step was carried out at $72^{\circ} \mathrm{C}$ for $5 \mathrm{~min}$. Each sample at each locus was amplified three times and an allele was accepted when it was detected at least twice.

DNA extraction and PCR amplification were conducted in separate rooms and positive and negative controls were included in all the experiments.

PeerJ reviewing PDF | (2017:02:16039:1:1:NEW 25 Apr 2017) 
111

112

113

114

115

116

117

118

119

120

121

122

123

124

125

126

127

128

129

130

131

\section{Data analysis}

Comparison of storage type and storage time

The mean microsatellite amplification success was the proportion of all the microsatellite loci that were successfully

amplified for each storage type and each storage time. We further analyzed the genotyping errors for microsatellites;

these were the allelic dropouts (ADO, one allele of a heterozygote was lost during the amplification) and the false

alleles (FA, the allele generated during PCR due to a slippage artifact). We determined the occurrence of ADO and

FA by comparing the genotype produced by the fecal samples with the genotype obtained from the blood samples.

$\mathrm{ADO}$ and FA rates were calculated according to the equations 2 and 4 described in Broquet \& Petit (2004).

We analyzed three dependent variables: amplification success, ADO, and FA rates using a repeated measure general linear model, with preservation methods as the "between-subject variable" and storage time as the "repeated element followed by a Tukey's multiple comparison test. If the effects between the factors were significant, we interpreted simple main effects using least significant differences analysis to separate the means.

Comparison of fragment length and repeat motif at microsatellite loci

We classified nine microsatellite loci by their fragment lengths and obtained 3 grades: Grade I included Gp4 and Panda25, which had a product size smaller than 150 bp; Grade II contained Panda29 and Gpl29, with product sizes between 150 and 200 bp; Grade III had Aime10, Aime16, Panda22, Gpz6, and Gpl60 with product sizes between 200 and 300 bp. Moreover, the loci were classified into 3 groups with di-(Gp4, Aime10, and Aime16), tri- (Panda22, Panda25, and Panda29), and tetra-nucleotide repeats (Gpz6, Gpl29, and Gpl60), respectively. We compared the effects of fragment length and repeat motif on the amplification success, and the ADO and FA rates using a multivariate general linear model with the amplification success, ADO, and FA rates as dependent variables, fragment length and repeat motif as fixed factors, and storage type as covariate. We adopted the data obtained at the 
132 133

storage time of one month to avoid the time effect. All the statistical tests were performed using SPSS 20.0 (IBM, Chicago, USA).

\section{Results}

\section{Evaluation of storage type and storage time on the amplification success at microsatellite}

The storage type had an effect on the microsatellite amplification success $\left(\mathrm{F}_{4,80}=9.976, P<0.001\right)$. The samples stored in $\mathrm{EtOH}$ showed the best performance (89.1\%), followed by those stored in the 2-step storage medium (87.6\%), DET/EtOH $/-20^{\circ} \mathrm{C}(80.4 \%)$, and at $-20^{\circ} \mathrm{C}(57.8 \%$; Fig. 1A). The amplification success in the freezing type was significantly lower than in the other four storage types and there were no significant differences between any other comparisons (Table S1).

We observed that storage time had an effect on the amplification success, as well $\left(\mathrm{F}_{2,80}=61.306, P<0.001\right)$. As the storage time increased, the amplification success decreased from $90.7 \%$ to $68.0 \%$ or approximately by $23 \%$ (Fig. 1B). Amplification successes obtained from three storage times were different from each other (Table S2).

There was an interaction between the effects of the storage time and storage types on the microsatellite amplification success $\left(\mathrm{F}_{8,80}=9.099, P<0.001\right)$, revealing that the optimal storage type varied among the different storage times (Fig. 1C). The highest amplification success in the first month was obtained from EtOH and 2-step storage medium (92.8\%). The 2-step storage medium and EtOH showed the best performance in the samples stored for 3 months $(90.8 \%)$ and 6 months $(87.6 \%)$, respectively. The amplification success in the samples stored under freezing conditions was least at all the three storage times (Fig. 1C). 
151

152

153

154

155

156

157

158

159

160

161

162

163

164

among the five storage types when the samples were stored for 3 months $\left(\mathrm{F}_{4,80}=9.590, P<0.001\right)$ and 6 months $\left(\mathrm{F}_{4,80}=18.970, P<0.001\right)$, but not in those stored for 1 month $\left(\mathrm{F}_{4,80}=0.240, P=0.915\right)$. Specifically, at the end of the 3 months, the amplification success of the freezing type was significantly lower than the other four storage types and the other four types did not show any statistical difference (Table S1). As the storage time increased to 6 months, the amplification success in the freezing type was still significantly lower than in the other four storage types, and EtOH storage had a significantly higher amplification success than the EtOH $/-20^{\circ} \mathrm{C}, \mathrm{DET}$, and $-20^{\circ} \mathrm{C}$ storage, but not the 2-step storage (Table S1). There were no significant differences between any other comparisons (Table S1).

\footnotetext{
We also compared the amplification success among the three storage times for each storage type (Table S2).
}

There was significant difference in the amplification success between the storage times when the samples were stored in $\mathrm{EtOH} /-20^{\circ} \mathrm{C}\left(\mathrm{F}_{2,32}=9.276, P=0.001\right)$, DET $\left(\mathrm{F}_{2,32}=23.543, P<0.001\right)$ and $-20^{\circ} \mathrm{C}\left(\mathrm{F}_{2,32}=37.535, P<\right.$ $0.001)$, but not in $\operatorname{EtOH}\left(\mathrm{F}_{2,32}=1.233, P=0.305\right)$ and 2 -step storage $\left(\mathrm{F}_{2,32}=2.408, P=0.092\right)$. This signified the variation in the decline of the amplification success among the different storage types. The amplification success in the samples stored at $-20^{\circ} \mathrm{C}$ decreased by $60 \%$ from 1 month to 6 months; for those stored in $\mathrm{DET}$, EtOH/-20 ${ }^{\circ} \mathrm{C}, 2-$ step storage medium, and EtOH, it decreased by 27.6, 20, 15, and 6\%, respectively.

\section{Evaluation of storage type and storage time on genotyping errors at microsatellites}

None of the three terms, the storage type, storage time, and the interaction between them, had an effect on the FA rates (storage time: $\mathrm{F}_{2,80}=1.365, P=0.261$; storage type: $\mathrm{F}_{4,80}=0.360, P=0.836$; storage time $\times$ storage type: $\mathrm{F}_{8,80}$ $=0.716, P=0.677)$. With respect to the ADO rate, only the storage type was significant (storage time: $\mathrm{F}_{2,80}=3.066$, $P=0.052$; storage type: $\mathrm{F}_{4,80}=6.435, P<0.001$; storage time $\times$ storage type: $\left.\mathrm{F}_{8,80}=1.127 P=0.354\right)$. Specifically for the storage type, the samples stored in $\mathrm{EtOH}$ showed the lowest $\mathrm{ADO}$ rate, followed by $\mathrm{EtOH} /-20^{\circ} \mathrm{C}, 2-\mathrm{step}$ 
171

172

173

174

175

176

storage medium, DET, and $-20^{\circ} \mathrm{C}$ (Table 1). The ADO rate in the freezing type was significantly higher than in the other four storage types and the other four storage types showed no significant differences among them (Table S3).

\section{Evaluation of fragment length and repeat motif on amplification success and genotyping errors at the} microsatellites

Fragment length had no effect on microsatellite amplification success, ADO and FA rates (amplification success:

$\mathrm{F}_{2,37}=2.057, P=0.142 ; \mathrm{ADO}: \mathrm{F}_{2,37}=0.349, P=0.707 ; \mathrm{FA}: \mathrm{F}_{2,37}=2.343, P=0.110$, Table S4).

The effect of repeat motif on the amplification success was not significant whereas its effects on the ADO and FA rates were significant (amplification success: $\mathrm{F}_{2,37}=0.326, P=0.724 ; \mathrm{ADO}: \mathrm{F}_{2,37}=8.468, P=0.001 ; \mathrm{FA}: \mathrm{F}_{2,37}=$ 45.347, $P<0.001$, Fig. 2). Tri-and tetra-nucleotide loci showed lower ADO and FA rates than di-nucleotide loci $(P$ $<0.01$ in both cases, Fig. 2, Table S5) whereas the FA rate and ADO rate from tri-and tetra-nucleotide loci were similar $(P>0.05$ in both the cases, Fig. 2, Table S5).

There was no interaction between the effects of the fragment length and repeat motif on the microsatellite amplification success, ADO, and FA rates (amplification success: $\mathrm{F}_{2,37}=2.742, P=0.078 ; \mathrm{ADO}: \mathrm{F}_{2,37}=0.945, P=$ $\left.0.398 ; \mathrm{FA}: \mathrm{F}_{2,37}=2.166, P=0.129\right)$.

\section{Discussion}

\section{Evaluation of the storage type and storage time}

In the present study, we found that both the sample storage type and storage time influenced the microsatellite amplification and the storage type affected the genotyping reliability. 
Methods for attempting to prevent degradation of fecal DNA samples include storing them in the preservation medium containing high salt concentration buffer, quick desiccation, or keeping the samples under low temperature conditions (reviewed in Tende et al. 2014). The present study showed that low temperature $\left(-20^{\circ} \mathrm{C}\right)$ was the least effective method for preserving the giant panda fecal DNA compared to the quick desiccation (EtOH and 2-step storage), high salt concentration (DET), and a combination of quick desiccation and low temperature $\left(\mathrm{EtOH} /-20^{\circ} \mathrm{C}\right)$ methods, owing to lowest microsatellite amplification success and highest ADO rate $(P<0.05)$. Freezing was also found to be the least appropriate method in studies of Eurasian Badger (Frantz et al. 2003), black and sun bear (Wasser et al. 1997), wolf (Santini et al. 2007), and baboons (Frantzen et al. 1998). We did not dry the samples prior to $-20^{\circ} \mathrm{C}$ preservation; therefore, the high moisture in the samples might have speeded the degradation in our study.

No significant differences were observed among the other four storage types, i.e., EtOH, EtOH/-20 ${ }^{\circ} \mathrm{C}, 2$-step storage medium, and DET, based on the average amplification success and FA and ADO rates $(P>0.05)$. The samples stored in EtOH showed the highest microsatellite amplification success but the lowest ADO and FA rates. Similar to our results, EtOH scored the highest in the study on brown bear (Murphy et al. 2002), Eurasian badger (Frantz et al. 2003), lion (Tende et al. 2014), or was equally high as the other methods in the study on primate scats (Whittier et al. 1999), gorilla (Roeder et al. 2004), and coyote (Panasci et al. 2011), although the preservation methods evaluated in these studies were different. These findings revealed that EtOH was a good preservation buffer for fecal samples, irrespective of the diet of the species and this method could be used in either tropical or temperate weather (Roeder et al. 2004; Tende et al. 2014).

As observed in the present study, the 2-step storage also worked well in the preservation of giant panda fecal samples based on slightly lower amplification success (1\%) and slightly higher ADO rate (3\%) relative to the EtOH- 
209

210

211

212

213

214

215

216

217

218

219

220

221

222

223

224

225

226

227

preserved samples (Fig. 1; Table 1; Table S1; Table S3). Similar to our results, Tende et al. (2004) reported that the lion feces stored in the 2-step storage medium had lower amplification success rate than that preserved in EtOH. The

2-step storage medium was a combination of $\mathrm{EtOH}$ and silica, which was soaked in $\mathrm{EtOH}$ for a short period prior to desiccation by silica. We could not comment on the effectiveness of silica alone in storing the giant panda fecal samples compared to the 2-step storage medium owing to the lack of data on silica preserved samples in our study.

However, in the studies on gorilla and chimpanzee feces (Nsubuga et al. 2004; Roeder et al. 2004) and the tiger feces (Reddy et al. 2012), the 2-step storage medium was superior to silica alone for producing more DNA, but outperformed or acted equally well as EtOH. The high-quality amplification obtained from the samples preserved in the 2-step storage medium might be attributed to two types of desiccation. We presumed that the 2-step storage medium would perform better than the silica alone in preserving the giant panda fecal DNA since EtOH was more effective than this medium in desiccating the samples in our case.

The other two storage types, $\mathrm{EtOH}$ at $-20^{\circ} \mathrm{C}$ and DET, would not be recommended for microsatellite fecal studies in giant panda, because of the great decrease in the amplification success during the six-month storage. Although it was revealed that EtOH at $-20^{\circ} \mathrm{C}$ (Santini et al. 2007) and DET (Frantz et al. 2003; Frantzen et al. 1998; Panasci et al. 2011) worked best in previous studies, these studies did not evaluate the variation of long term storage for these methods. Thus, we believe that these two methods might be useful for short- term fecal sample preservation. Fecal samples preserved in $\mathrm{EtOH}$ at $-20^{\circ} \mathrm{C}$ performed worse than those preserved in $\mathrm{EtOH}$ with the storage time increased.

We speculated that the variation in the temperature (repeated freezing and thawing) might have led to the DNA degradation, which was supported by other studies (Ross et al. 1990; Shao et al. 2012). 
228

229

230

231

232

233

234

235

236

237

238

239

240

241

242

243

244

245

246

Like in other studies (Murphy et al. 2002; Santini et al. 2007; Soto-Calderon et al. 2009), we found that the storage time had a negative effect on the microsatellites amplification success, suggesting that the preservation medium could only retard but not stop the degradation of fecal DNA by endonucleases. We recommended that fecal DNA should be extracted as soon as the samples are collected in order to obtain high DNA quality, to increase the accuracy of microsatellite genotyping, and to decrease the associated cost. Furthermore, our results indicated that the greatest decrease in microsatellite amplification success began at 3 months, but as we lacked data on genotyping from different months, we could not evaluate the relationship between the degradation rate and the storage time.

Overall, it was indicated from our study that the samples stored in EtOH and 2-step storage medium were more stable than those stored in $\mathrm{EtOH} /-20^{\circ} \mathrm{C}$, DET, and at $-20^{\circ} \mathrm{C}$, since they had a slight change in microsatellite amplification success among the three storage times whereas the amplification success in the other three storage types decreased greatly with time. Thus, for microsatellite studies using samples stored for longer periods, EtOH, and 2-step storage medium might be a better choice.

\section{Evaluation of fragment length and repeat motif for microsatellites}

For microsatellite studies, apart from selecting an appropriate storage type, careful choice of microsatellite loci could optimize the amplification and genotyping success of the fecal samples (reviewed in Broquet et al. 2007).

Broquet et al. (2007) found that amplification success decreased with the fragment length for microsatellites

whereas the genotyping error rate increased. In the present study, we did not detect a linear relationship between the fragment length and amplification success or genotyping error rates, which might be due to the limited number of loci analysed by us. 
247 Furthermore, it was reported that shorter repeat units produced lower ADO rates relative to the longer ones

248 (Broquet et al. 2007), which was opposite to our results. Our findings revealed that the highest FA rates were also

249 obtained from the di-nucleotide microsatellite loci. Broquet et al. (2007) did not summarize the relationship between

250 the repeat motif and FA rate owing to lack of data. False alleles were mostly caused in the process of auto-calling;

251 therefore, it was normal that FA rate of di-nucleotide loci was higher because they were prone to slippage during

252 PCR, which caused misscoring (Pompanon et al. 2005). Based on our findings, we recommended that it would be

better to choose microsatellites with longer repeat motifs in fecal studies to obtain higher genotyping success.

\section{Conclusion}

The findings of the present study revealed that fecal storage method should be carefully chosen in microsatellite

\section{Acknowledgments}


267 We thank Wei M for the suggestion of fecal samples collection, Deng XX for blood collection, Liu Y, Dong JH,

268 Xiong LB, Xu X, Liu HT, Wang YF, Tang C, Zheng YM, and Liu HT for the fecal samples collection, and Dr. He K

269 for reviewing the manuscript.

\section{References}

271

272

273

274

276

277

278

279

280

281

282

Broquet T, Ménard N, and Petit E. 2007. Noninvasive population genetics: a review of sample source, diet, fragment length and microsatellite motif effects on amplification success and genotyping error rates. Conservation Genetics 8:249-260. DOI: 10.1007/s10592-006-9146-5.

Broquet T, and Petit E. 2004. Quantifying genotyping errors in noninvasive population genetics. Molecular Ecology 13:3601-3608. DOI: 10.1111/j.1365-294X.2004.02352.x.

Chen YY, Zhu Y, Wan QH, Lou JK, Li WJ, Ge YF, and Fang SG. 2013. Patterns of Adaptive and Neutral Diversity Identify the Xiaoxiangling Mountains as a Refuge for the Giant Panda. PLoS ONE 8:e70229. DOI: 10.1371/journal.pone.0070229.

Constable JL, Ashley MV, Goodall J, and Pusey AE. 2001. Noninvasive paternity assignment in Gombe chimpanzees. Molecular Ecology 10:1279-1300. DOI: 10.1046/j.1365-294X.2001.01262.x.

Dalen L, Gotherstrom A, and Angerbjorn A. 2004. Identifying species from pieces of faeces. Conservation Genetics 5:109-111. DOI: 10.1023/b:coge.0000014060.54070.45.

Eggert LS, Eggert JA, and Woodruff DS. 2003. Estimating population sizes for elusive animals: the forest elephants of Kakum National Park, Ghana. Molecular Ecology 12:1389-1402. DOI: 10.1046/j.1365294X.2003.01822.x. 
286

287

288

289

290

291

292

293

294

295

296

297

298

299

300

301

302

303

304

Frantz AC, Pope LC, Carpenter PJ, Roper TJ, Wilson GJ, Delahay RJ, and Burke T. 2003. Reliable microsatellite genotyping of the Eurasian badger (Meles meles) using faecal DNA. Molecular Ecology 12:1649-1661.

DOI: 10.1046/j.1365-294X.2003.01848.x.

Frantzen MAJ, Silk JB, Ferguson JWH, Wayne RK, and Kohn MH. 1998. Empirical evaluation of preservation methods for faecal DNA. Molecular Ecology 7:1423-1428. DOI: 10.1046/j.1365-294x.1998.00449.x.

Garnier JN, Bruford MW, and Goossens B. 2001. Mating system and reproductive skew in the black rhinoceros. Molecular Ecology 10:2031-2041. DOI: 10.1046/j.0962-1083.2001.01338.x.

Huang J, Li YZ, Du LM, Yang B, Shen FJ, Zhang HM, Zhang ZH, Zhang XY, and Yue BS. 2015. Genome-wide survey and analysis of microsatellites in giant panda (Ailuropoda melanoleuca), with a focus on the applications of a novel microsatellite marker system. BMC Genomics 16. DOI: 10.1186/s12864-015-1268-z.

Huber S, Bruns U, and Arnold W. 2002. Sex determination of red deer using polymerase chain reaction of DNA from feces. Wildlife Society Bulletin 30:208-212.

Kohn MH, and Wayne RK. 1997. Facts from feces revisited. Trends in Ecology \& Evolution 12:223-227. DOI: 10.1016/s0169-5347(97)01050-1.

Kruglyak S, Durrett RT, Schug MD, and Aquadro CF. 1998. Equilibrium distributions of microsatellite repeat length resulting from a balance between slippage events and point mutations. Proceedings of the National Academy of Sciences of the United States of America 95:10774-10778. DOI: 10.1073/pnas.95.18.10774.

Murphy MA, Waits LP, Kendall KC, Wasser SK, Higbee JA, and Bogden R. 2002. An evaluation of long-term preservation methods for brown bear (Ursus arctos) faecal DNA samples. Conservation Genetics 3:435- 
440. DOI: 10.1023/a:1020503330767.

306

307

308

309

Nsubuga AM, Robbins MM, Roeder AD, Morin PA, Boesch C, and Vigilant L. 2004. Factors affecting the amount of genomic DNA extracted from ape faeces and the identification of an improved sample storage method. Molecular Ecology 13:2089-2094. DOI: 10.1111/j.1365-294X.2004.02207.x.

Panasci M, Ballard WB, Breck S, Rodriguez D, Densmore LD, III, Wester DB, and Baker RJ. 2011. Evaluation of fecal DNA preservation techniques and effects of sample age and diet on genotyping success. Journal of Wildlife Management 75:1616-1624. DOI: 10.1002/jwmg.221.

Piggott MP, and Taylor AC. 2003. Extensive evaluation of faecal preservation and DNA extraction methods in Australian native and introduced species. Australian Journal of Zoology 51:341-355. DOI: 10.1071/zo03012.

Pompanon F, Bonin A, Bellemain E, and Taberlet P. 2005. Genotyping errors: causes, consequences and solutions. Nature Reviews Genetics 6:847-859. DOI: 10.1038/nrg1707.

Reddy PA, Bhavanishankar M, Bhagavatula J, Harika K, Mahla RS, and Shivaji S. 2012. Improved methods of carnivore faecal sample preservation, DNA extraction and quantification for accurate genotyping of wild tigers. PLoS ONE 7:e46732. DOI: 10.1371/journal.pone.0046732.

Roeder AD, Archer FI, Poiner HN, and Morin PA. 2004. A novel method for collection and preservation of faeces for genetic studies. Molecular Ecology Notes 4:761-764. DOI: 10.1111/j.1471-8286.2004.00737.x.

Ross KS, Haites NE, and Kelly KF. 1990. Repeated freezing and thawing of peripheral blood and DNA in suspension: effects on DNA yield and integrity. Journal of Medical Genetics 27:569-570. 
324 Santini A, Lucchini V, Fabbri E, and Randi E. 2007. Ageing and environmental factors affect PCR success in wolf

325

326

327

328 (Canis lupus) excremental DNA samples. Molecular Ecology Notes 7:955-961. DOI: 10.1111/j.14718286.2007.01829.x.

Shao W, Khin S, and Kopp WC. 2012. Characterization of effect of repeated freeze and thaw cycles on stability of genomic DNA using pulsed field gel electrophoresis. Biopreservation \& Biobanking 10:4.

Shen F, Watts PC, He W, Zhang Z, Zhang A, Sanderson S, Kemp SJ, and Yue B. 2007. Di-, tri- and tetranucleotide microsatellite loci for the giant panda, Ailuropoda melanoleuca. Molecular Ecology Notes 7:1268-1270. DOI: $10.1111 / \mathrm{j} .1471-8286.2007 .01851 . x$.

Soto-Calderon ID, Ntie S, Mickala P, Maisels F, Wickings EJ, and Anthony NM. 2009. Effects of storage type and time on DNA amplification success in tropical ungulate faeces. Molecular Ecology Resources 9:471-479. DOI: $10.1111 / \mathrm{j} .1755-0998.2008 .02462 . x$.

Taberlet P, Griffin S, Gossens B, Questiau S, Manceau V, Escaravage N, Waits LP, and Bouvet J. 1996. Reliable genotyping of samples with very low DNA quantities using PCR. Nucleic Acids Research 24:3189-3194.

DOI: $10.1093 /$ nar/24.16.3189.

Tende T, Hansson B, Ottosson U, and Bensch S. 2014. Evaluating preservation medium for the storage of DNA in African lion Panthera leo faecal samples. Current Zoology 60:351-358.

Wan QH, Zhu L, Wu H, and Fang SG. 2006. Major histocompatibility complex class II variation in the giant panda (Ailuropoda melanoleuca). Molecular Ecology 15:2441-2450. DOI: 10.1111/j.1365-294X.2006.02966.x. 
methods to field studies of Ursids. Molecular Ecology 6:1091-1097. DOI: 10.1046/j.1365294X.1997.00281.x.

Whittier CA, Dhar AK, Stem C, Goodall J, and Alcivar-Warren A. 1999. Comparison of DNA extraction methods for PCR amplification of mitochondrial cytochrome c oxidase subunit II (COII) DNA from primate fecal samples. Biotechnology Techniques 13:771-779. DOI: 10.1023/a:1008956921159.

Wu H, Zhan XJ, Zhang ZJ, Zhu LF, Yan L, Li M, and Wei FW. 2009. Thirty-three microsatellite loci for noninvasive genetic studies of the giant panda (Ailuropoda melanoleuca). Conservation Genetics 10:649652. DOI: $10.1007 / \mathrm{s} 10592-008-9599-9$.

Zhan X, Zhang Z, Wu H, Goossens B, Li M, Jiang S, Bruford M, and Wei F. 2007. Molecular analysis of dispersal in giant pandas. Molecular Ecology 16:3792-3800. DOI: 10.1111/j.1365-294X.2007.03450.x.

Zhang B, Li M, Zhang Z, Goossens B, Zhu L, Zhang S, Hu J, Bruford MW, and Wei F. 2007. Genetic viability and population history of the giant panda, putting an end to the "evolutionary dead end"? Molecular Biology and Evolution 24:1801-1810. DOI: 10.1093/molbev/msm099.

Zhang BW, Wei FW, Li M, and Lu XP. 2004. A simple protocol for DNA extraction from faeces of the giant panda and lesser pandas. Acta Zoologica Sinica 50:452-458.

Zhang HM, Guo Y, Li DS, Wang PY, and Fang SG. 2009. Sixteen novel microsatellite loci developed for the giant panda (Ailuropoda melanoleuca). Conservation Genetics 10:589-592. DOI: 10.1007/s10592-008-9576-3.

Zhang W, Yie S, Yue B, Zhou J, An R, Yang J, Chen W, Wang C, Zhang L, Shen F, Yang G, Hou R, and Zhang Z. 2012. Determination of Baylisascaris schroederi Infection in Wild Giant Pandas by an Accurate and 
363 Zhu Y, Wan QH, Yu B, Ge YF, and Fang SG. 2013. Patterns of genetic differentiation at MHC class I genes and 364 microsatellites identify conservation units in the giant panda. BMC Evolutionary Biology 13:227. DOI: $10.1186 / 1471-2148-13-227$. 


\section{Figure captions}

367 Fig 1 Rate of amplification success at giant panda microsatellite loci amplified from fecal DNA stored in five

368 storage types at three storage intervals. The average amplification success across the three storage times and across

369 the five storage types is depicted in the upper space of each figure. The whiskers show the values of the minimum,

$3702.5^{\text {th }}$ percentile, median, and $97.5^{\text {th }}$ percentile

371 Fig 2 Average amplification success rate (a), allelic dropout (b), and false allele rates (c) for di-, tri-, tetra-nucleotide

372 loci obtained from giant panda fecal DNA. The whiskers show the values of the minimum, $2.5^{\text {th }}$ percentile, median,

373 and $97.5^{\text {th }}$ percentile 
374 Table 1 Allele dropout and false allele rates over nine microsatellite loci among the five storage types in samples

375 preserved for 1,3 , and 6 months

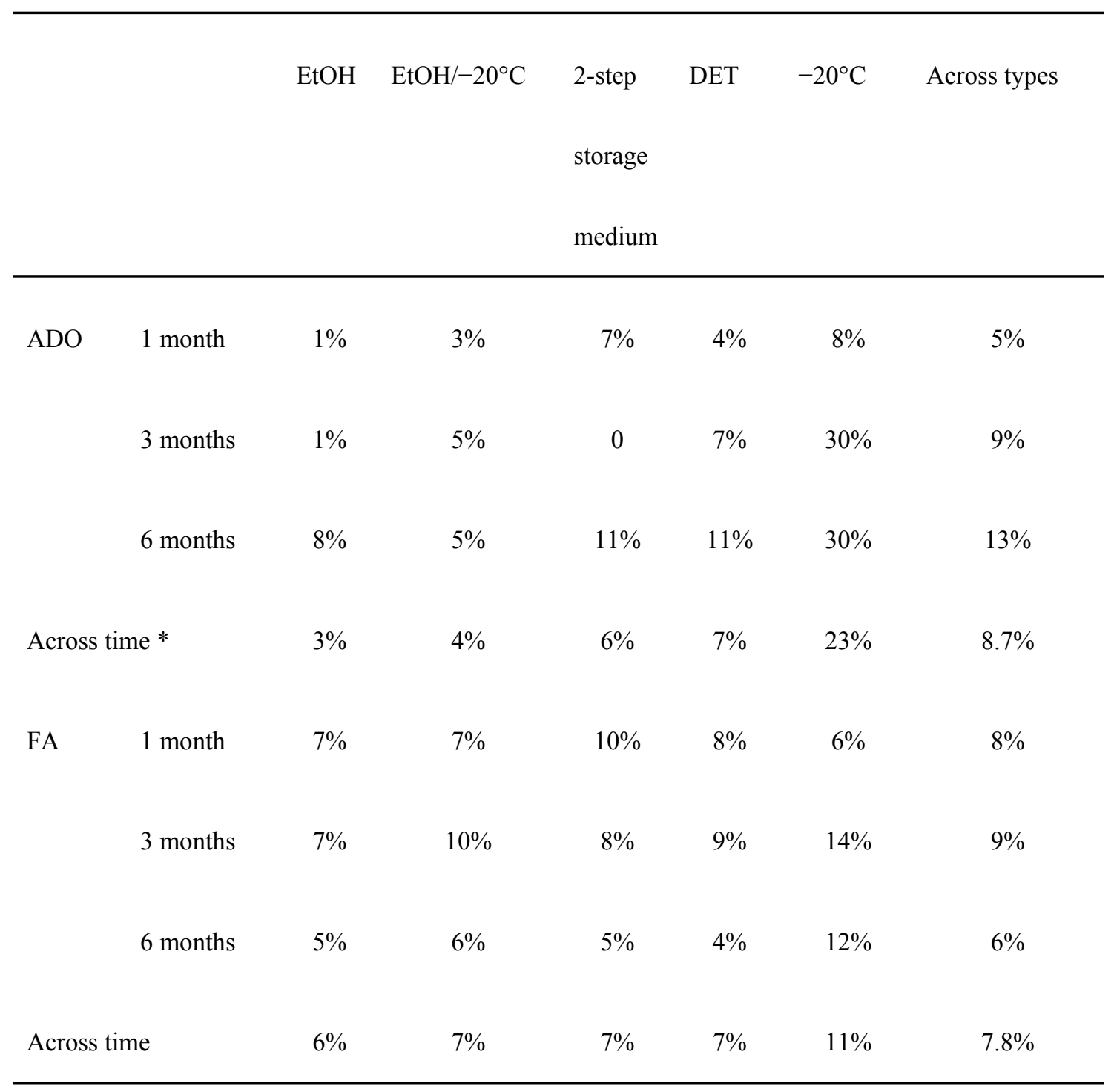

376 Note: ADO and FA are the abbreviations for allele dropout and false allele, respectively. Asterisk denotes that the storage type had an effect on the average amplification success over time 


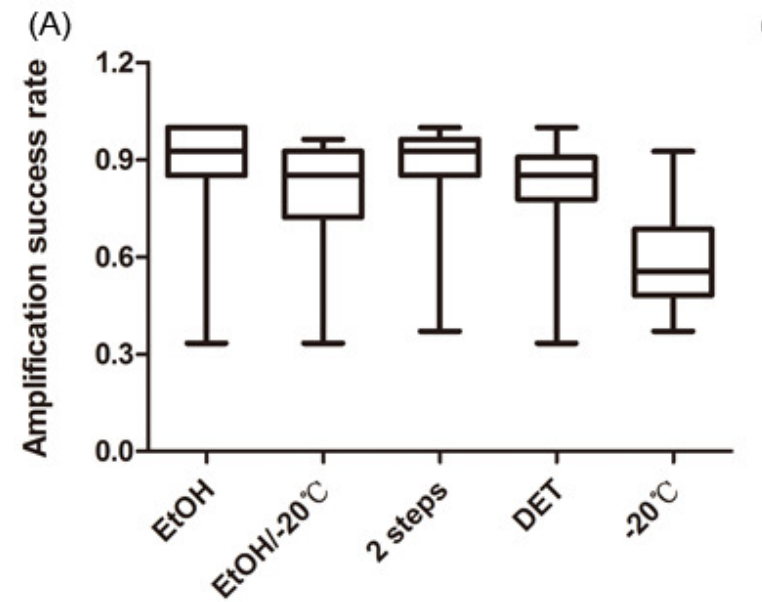

(B)

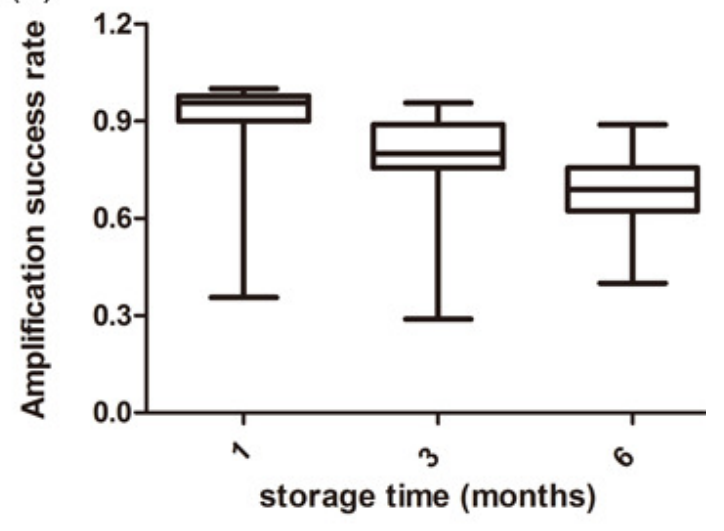

(C)

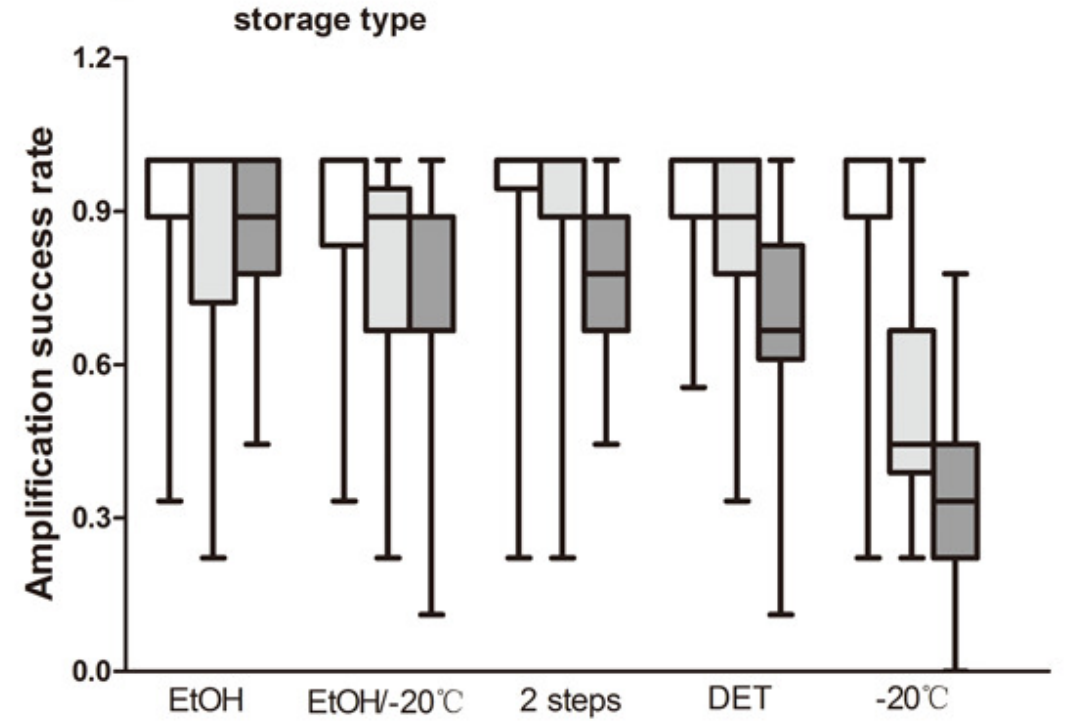

storage type 

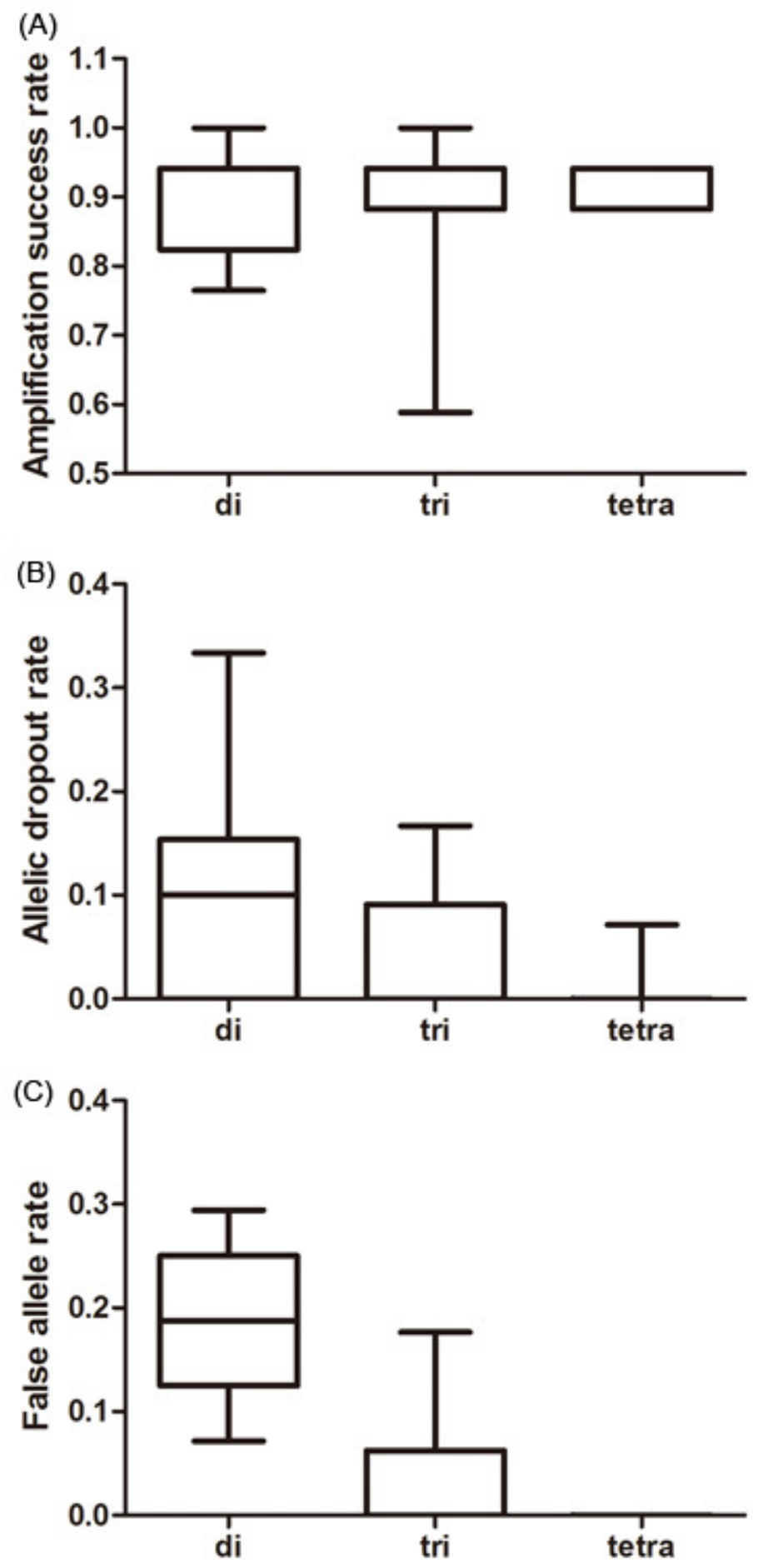